\title{
La nueva legislación uruguaya sobre marihuana y sus derivados
}

New Uruguayan law on cannabis and its derivatives

\section{Fernando Rovira}

Abogado, Doctor en Derecho y Ciencias Sociales. Profesor del Derecho del Trabajo y la Seguridad Social, Universidad de la Republica y Universidad de la Empresa Uruguay; profesor de Derecho Sanitario UCUDAL; profesor de Derecho de la Salud Ocupacional. Abogado Asesor del Vice Ministro de Salud Pública, Ministerio de Salud Pública. Montevideo, Uruguay.

\section{Juan Pablo Decia}

Abogado, Doctor en Derecho y Ciencias Sociales. Asesor Legal del Director del Hospital Maciel. Montevideo, Uruguay.

\section{Gonzalo La Rosa}

Asesor del Vice Ministro de Salud Pública y Representante del Ministerio de Salud Publica en la Junta Nacional de Drogas. Montevideo, Uruguay.

Ley $n^{\circ} 19.172$, de 20 de diciembre de 2013. (Publicada en el Diario Oficial de 7 de enero de 2014).

http://www.parlamento.gub.uy/leyes/AccesoTextoLey.asp?Ley=19172\&Anchor=

\section{Génesis de la ley}

La iniciativa de legislar sobre el control y regulación por parte del Estado de la importación, producción, adquisición, almacenamiento, comercialización y distribución de marihuana surgió en el Gabinete de Seguridad que integran diversos Ministerios de Gobierno, dentro de un paquete de medidas y en el marco de la denominada "Estrategia por la vida y la convivencia".

Estadísticas de la Junta Nacional de Drogas revelan que la marihuana es la droga ilegal de mayor consumo en el país, solo superada por dos sustancias psicoactivas legales, el alcohol y el tabaco, por lo que el mercado de cannabis y sus derivados era un dato de la realidad, insoslayable y anterior al dictado de la nueva legislación.

Se trata de un mercado dominado y regulado exclusivamente por el narcotráfico, que impone sus reglas "a sangre y fuego" y que coloca a los 
consumidores y a la sociedad en su conjunto en situación de extrema vulnerabilidad y de exposición a riesgos muy superiores a los del simple consumo de la sustancia.

La legislación anterior no penaba el consumo de sustancias psicoactivas sino la producción, el almacenamiento y la comercialización, por lo cual, para acceder a la marihuana, los consumidores estaban obligados a cometer un delito al concurrir al mercado negro del narcotráfico, aumentando los riesgos a los que están expuestos. Se trataba de una contradicción perversa: consumo permitido-acceso prohibido.

El "efecto góndola" refiere al supermercado de drogas del narcotráfico, regulado por sus propias leyes, que les permite tanto ampliar la oferta como desabastecer algunos "artículos" para imponer otros, de acuerdo a sus intereses económicos. Su consumo ya estaba presente; no se crea un mercado, sino que se asume la regulación de uno pre-existente, con la ley habilitando el acceso a la sustancia y alejando al usuario del narco-mercado.

\section{El fracaso de la estrategia represiva}

La estrategia de "guerra a las drogas", sostenida hasta ahora en la mayoría de los países del planeta, ha sido sistemáticamente derrotada. La realidad se ha ocupado de demostrar que es errónea y que lo único que con ello se logra es agravar el problema que se pretendía resolver.

Puede calificarse de derrota "militar" en sentido estricto: alimenta conflictos bélicos en muchos países del mundo y de la región y coloca al narcotráfico, con contingentes numerosos y en muchos casos mejor pertrechados que los del Estado, en situación de ventaja estratégica.

El reflejo de la lógica bélica a nivel nacional se expresa en la insistencia de los sectores más conservadores en reclamar la prohibición y la represión como única respuesta, y en la arbitraria asociación joven-droga-delito que identifica de manera oportunista el uso de sustancias y los problemas de seguridad pública.

El descrédito de las políticas y andamiajes jurídicos represivos a nivel mundial ha puesto en tela de juicio, definitivamente, esa forma de abordar el problema y, desde sectores de la sociedad civil, se insiste sobre la necesidad de un cambio de paradigma en el abordaje de la cuestión. 


\section{La nueva legislación uruguaya}

El Uruguay ha sido el primer país en pasar de lo declarativo a la acción, presentando una iniciativa integral y de carácter nacional que retoma el papel central del Estado. Sin abandonar la función represiva - hacia el narcotráfico, no hacia los usuarios-, concibe el uso de sustancias psicoactivas como un problema de salud pública que exige, por lo tanto, un abordaje predominantemente sanitario.

El proceso de discusión de la ley fue altamente participativo, de modo que el artículo único - con el que el Poder Ejecutivo envió la iniciativa al Parlamento, como puntapié inicial provocador de intensos debates en el seno de la sociedad civil, de los partidos políticos y del propio Parlamento, que consultó a la academia, a organizaciones sociales de usuarios, a instituciones públicas y privadas con competencia en la materia etc., todo dentro del libre juego de la democracia, cristalizó en la aprobación de una ley de 44 artículos, omnicomprensiva de los diversos procesos de control y regulación del uso del cannabis, ahora a cargo del Estado.

Los aspectos sobresalientes de la nueva ley son:

\section{Estrategia de Reducción de Riesgos y Daños (RRDD)}

La aludida perspectiva sanitaria aparece ya en el Art. 1 de la ley que declara "...de interés público las acciones tendientes a proteger, promover y mejorar la salud pública de la población mediante una política orientada a minimizar los riesgos y a reducir los daños del uso de cannabis...".

La ley define entonces no solo la "tesitura sanitarista", sino cuál es el marco conceptual de la política pública: la reducción de riesgos y daños (RRDD). Los arts. 3 , 4 y 9 a 15 insisten en diversos aspectos vinculados a la RRDD:

- el derecho de todas las personas al disfrute del más alto nivel posible de salud, al disfrute de los espacios públicos en condiciones seguras y a las mejores condiciones de convivencia;

- la protección de los habitantes ante los riesgos inherentes al comercio ilegal, el narcotráfico y el crimen organizado;

- la educación, concientización y prevención de los riesgos para la salud derivados del uso del cannabis; 
- la adopción de políticas e implementación de dispositivos para la promoción de la salud, la prevención del uso problemático de cannabis y el asesoramiento, orientación y tratamiento de usuarios problemáticos;

- la prohibición de toda publicidad, promoción, auspicio o patrocinio de productos de cannabis psicoactivo;

- la prohibición del acceso al cannabis psicoactivo con uso recreativo a los menores de edad (18 años);

- la inhabilitación para conducir vehículos cuando la concentración de tetrahidrocannabinol (THC) en el organismo supere la permitida por la reglamentación.

Con ésta concepción como eje, es que los gobiernos progresistas han desarrollado la políticas públicas referidas al uso de las sustancias psicoactivas más prevalentes (regulación del tabaco u de alcohol) y el Ministerio de Salud de Uruguay aborda en la actualidad la reglamentación de los aspectos sanitarios de la ley de regulación de marihuana.

Esta ley se integra al conjunto de políticas promovidas, con especial énfasis, en el segundo gobierno del Frente Amplio, creando normativa e institucionalidad nueva que generó notables avances en la agenda social. Éste ha sido un rasgo distintivo del gobierno del Presidente José Mujica, con altísimo reconocimiento nacional e internacional.

\section{Tres formas de acceso al cannabis}

Se sustituye el acceso a través del narcotráfico por algunas de las siguientes formas legales:

\section{a) Autocultivo}

Autoriza la plantación, el cultivo y la cosecha domésticos de plantas de cannabis de efecto psicoactivo para el consumo personal o compartido en el hogar:

- hasta 6 plantas y un máximo de 480 gramos anuales del producto de la recolección;

- registro de autocultivadores: identidad de las personas=datos sensibles.

\section{b) Clubes de membresia}

- De 15 a 45 socios;

- hasta 99 plantas y un máximo de acopio anual proporcional al número de socios; 
- con personería jurídica otorgada por el Poder Ejecutivo;

- registro de clubes: identidad de las personas=datos sensibles.

\section{c) Compra en farmacias}

- Que cuenten con licencia de expendio otorgada por el organismo rector;

- inscripción en Registro del usuario, en caso de uso recreativo: identidad de las personas $=$ datos sensibles;

- receta médica en caso de uso medicinal;

- no más de 40 gramos mensuales por usuario (la reglamentación, aún no dictada, prevé el fraccionamiento en 4 cajas de 10 gramos por semana).

\section{Organismo rector del sistema}

- Instituto de Regulación y Control del Cannabis (IRCA), bajo la Presidencia de la Junta Nacional de Drogas (JND) e integrado por representantes de ministerios con competencia en la materia.

Persona jurídica de derecho público no estatal.

Bajo el contralor del Poder Ejecutivo a través del Ministerio de Salud Pública.

Tres cometidos: regular la plantación, cultivo, cosecha, producción, elaboración, acopio, distribución y expendio de cannabis en todas las modalidades examinadas; promover acciones para la RRD; y fiscalizar el cumplimiento de la ley.

\section{Infracciones. Sanciones penales y económicas}

Apercibimiento, multa, destrucción, suspensión en registro, inhabilitación, clausura parcial o total, temporal o permanente de los establecimientos licenciatarios, todo según la gravedad de la infracción;

Sanciones penales en caso de actividades delictivas.

\section{Algunas respuestas ante cuestionamientos sinceros o tendenciosos}

Los detractores de la ley hablan de la "legalización" del consumo de marihuana y, por transitividad, del nuevo rol prescindente del Estado ante un estatuto de libertad, o de libertinaje, que agudiza el problema de la seguridad pública.

Ello no es así: la ley no "legaliza" el consumo sino que "regula" el mercado, sustrayéndoselo al narcotráfico y poniéndolo bajo el control del Estado que, lejos de ser prescindente, es intervencionista y corajudo. 
En el mismo sentido, es conveniente utilizar "usuarios de sustancias psicoactivas" para referir a los consumidores en general. Los términos "adictos", "drogadictos" o "drogodependientes" tienen una connotación peyorativa y, en todo caso, corresponde solo una minoría de los usuarios que desarrollan uso problemático o adicción.

Ante la pregunta de si la marihuana causa daños a la salud, la respuesta es indudable: el uso de todas las sustancias psicoactivas expone a riesgos y causan daños. Uruguay demostró con el tabaco que éstos pueden reducirse con la regulación (no con la prohibición, que no estuvo ni está planteada con el tabaco o con el alcohol). Los usuarios que concurran a la farmacia, que auto cultiven o que se asocien a clubes están expuestos a menores riesgos que quienes fuman los "ladrillos" que provienen del exterior y que la policía incauta, que contienen residuos de todo tipo que aumentan los daños. Garantizando la calidad, se disminuyen los daños producidos por su consumo (no los evita: los reduce).

Por otra parte, la propia ley los reconoce, y define políticas públicas de educación y salud orientadas a desestimular el consumo, obligando al Sistema de Salud a contar con los dispositivos de promoción, prevención, atención y rehabilitación de aquellos usuarios problemáticos.

El consumo de alcohol es el primer causante de daños sociales, apareciendo asociado a la violencia, en general, y a la violencia doméstica y contra la mujer, en particular, generando, además, enormes cargas económicas al sistema de salud. Sin embargo, en el imaginario popular su consumo está normalizado y no se percibe como riesgoso. Es preocupación del gobierno la baja percepción de riesgo que del consumo de marihuana tienen los jóvenes. Las políticas de promoción de hábitos saludables, de prevención, de educación etc., cuyo desarrollo exige la ley, están orientadas a aumentar la percepción de riesgo, así como a desestimular el consumo y a minimizar riesgos y daños en quienes han optado por su uso.

Los usuarios de pasta base (PBC) también usan marihuana es otra de las afirmaciones para cuestionar la ley. Efectivamente la gran mayoría de los usuarios de PBC (que son la gran minoría: $0.5 \%$ ) usan marihuana, y la usan sistemáticamente, para amortiguar los efectos de la "bajada" de la PBC. Lo contrario es falso, pero subyacente a la idea de la escalada y de otra afirmación predilecta:

La marihuana es la puerta de entrada a drogas más pesadas. La conceptualización de la escalada de drogas está íntimamente asociada a la visión 
bélica de "guerra a las drogas" y está tan perimida como ésta. Es la expresión de una visión centrada en la animación y demonización de la sustancia. "Decile NO a las drogas" es su slogan característico, y sus ofertas terapéuticas son de corte voluntarista, proponiendo la abstinencia como única alternativa, promoviendo la sustitución del vínculo adictivo con la sustancia por el de la comunidad religiosa que, en la gran mayoría de los casos, está detrás (o delante) de este tipo de alternativas. Reconociendo que, para algunos usuarios en particular, ésta pueda ser una opción válida, la política pública desarrollada por el gobierno y recogida en la ley que regula la marihuana concibe el uso de sustancias como un fenómeno complejo, centrado en los tipos de relación que los usuarios establecen con una sustancia (y no en la sustancia), y que solo en algunos casos (aproximadamente el 10\%) se vuelve problemático.

Más contundente aún es que la evidencia disponible lo desmiente: el $85 \%$ de los usuarios de drogas ilegales en nuestro país solo consume marihuana, según estadísticas de la Junta Nacional de Drogas.

El consumo adictivo de sustancias psicoactivas implica un uso problemático, pero solo una parte minoritaria de los usuarios de sustancias psicoactivas desarrolla adicción, que tiene componentes comunes con otros consumos adictivos, de ropa, de comida, de relaciones personales. Nuestro Presidente Mujica se ha expresado con profundidad en múltiples ocasiones acerca de éste asunto y, por ello y por su estilo de vida acorde con lo que predica, es conocido y reconocido en el mundo.

Registros. Todo usuario de marihuana queda habilitado por la ley para el auto cultivo, para asociarse con otros usuarios en un club, o para acceder a ésta en farmacias. En todos los casos, bajo límites claramente establecidos y registros a los que estarán obligados. Estos registros no son una política represiva sino de protección de estos ciudadanos, y su confidencialidad queda garantizada por la ley de Habeas Data. Un ciudadano usuario de marihuana registrado en cualquiera de los modos de acceso estará protegido de ser vinculado con el narcotráfico (en tanto no lo esté, por supuesto). Por el contrario, un auto cultivador que no se registrase estaría fuera de la ley, y sería eventualmente objeto de las sanciones previstas.

¿Se van a legalizar otras drogas? En general el consumo de sustancias psicoactivas debe ser regulado, pero no pueden regularse todas por igual. Las sustancias que se consumen con el objetivo de alterar la conciencia no son todas. El tabaco, por ejemplo, no se consume con esa finalidad. El alcohol puede ser 
consumido como alimento, sin el objetivo de alterar la conciencia, pero también consumido con ese fin; las prácticas en uno y otro caso son bien distintas y necesita una regulación específica. La marihuana no puede ser considerada nunca un alimento, cualquiera sea el hábito de consumo, y siempre se la consume con el fin de alterar la conciencia, por lo que también tiene especificidades que están recogidas en la ley y consideradas en la reglamentación. De todas maneras hoy no hay condiciones internacionales, regionales ni nacionales para ampliar la regulación al consumo de otras sustancias. Tabaco, marihuana y alcohol - con un proyecto de ley ya en discusión - son las tres sustancias de mayor prevalencia de consumo, muy por encima de las demás, con excepción de los psicofármacos.

\section{Las políticas de RRDD desde una perspectiva sanitaria}

La reducción de danos $(\mathrm{RD})$ parte de la base de que la abstinencia puede ser un objetivo poco realista o no deseable para algunos usuarios: sólo $10 \%$ está dispuesto o preparado para dejar de consumir en un momento dado. Entre un 60 y $80 \%$ no está dispuesto a consultar en ningún servicio de salud. Se trata, entonces, de qué podría ofrecerse al $90 \%$ restante. La abstinencia no es una condición en la RRDD y, menos aún, un valor moral a cuidar, aunque sí es un objetivo deseable, pero no el único: en muchos casos el objetivo es modificar hábitos, conductas o modalidades de consumo por otros que produzcan daños menores.

Reconoce que el uso de drogas tiene efectos en la salud psico-física, las relaciones, el trabajo y estudio, la economía doméstica, la ley. Estas consecuencias pueden impactar en distintos niveles: individual, relacional próximo, comunitario (vecindario, compañeros, colegas) y social (estructuras y funciones de la sociedad).

La RRDD enfatiza el respeto por las personas y el ayudarlos en todas las circunstancias a aumentar las probabilidades de mejorar su salud. Deriva en el esquema conceptual de la promoción de salud, siendo la RD una de las tantas estrategias para disminuir los riesgos y daños, promover la salud y evitar la enfermedad.

Riesgo: conductas o condiciones de uso de sustancias psicoactivas que han demostrado estar vinculadas a un daño. No debería ser aplicada a condiciones y situaciones propias de la vida sino a las conductas asumidas en esas situaciones. Suele decirse que la adolescencia es un factor de riesgo para el consumo de drogas, cuando, en realidad, el riesgo está en algunas de las conductas asumidas por los 
adolescentes y estimuladas desde el actual modelo de socialización, y no en su condición de tales.

Como otras intervenciones médicas, las estrategias de RD se definen en torno al principio de primum non nocere. La estigmatización promueve exclusión y marginalización, factores éstos que, sí, han sido demostrados como de riesgo para el desarrollo del uso problemático de drogas, tal y como ocurre como cuando, frente a una tenencia simple, estos jóvenes son recluidos junto a delincuentes y narcotraficantes. O como ocurre cuando son separados de sus actividades curriculares o laborales para ser internados.

Las políticas de RRDD tienen como objetivos generales:

- reducir la morbi-mortalidad;

- prevenir enfermedades infecto-contagiosas;

- mejorar la calidad de vida;

- incrementar la accesibilidad y el contacto con el sistema de salud.

Como objetivos específicos:

- diversificar la oferta asistencial;

- analizar nuevas modalidades terapéuticas;

- proporcionar respuestas inmediatas a nivel psico-social;

- proporcionar recursos y respuestas para poblaciones particularmente problemáticas (trabajadores sexuales, dependientes de larga evolución, usuarios con deterioro orgánico y personal, usuarios con trastornos mentales, población carcelaria).

Los programas de RRDD:

- aceptan como válidos los objetivos paliativos sin entrar en competencia con los curativos;

- deben ser amplios y accesibles;

- con objetivos graduales y personalizados surgidos del análisis de las necesidades individuales;

- construidos a partir de una relación dialogante con el usuario de drogas;

- orientados a incidir fundamentalmente en la modalidad de consumo. 